\title{
Endovascular Aneurysm Repair (EVAR) of an Infra-Renal Abdominal Aortic Aneurysm (AAA) in a Young Patient with Systemic Lupus Erythematosus (SLE)
}

Nick Zhi Peng Ng* and Chong Tze Tec

Singapore General Hospital, Singapore

\begin{abstract}
Aortic aneurysms are a rare cardiovascular complication of Systemic Lupus Erythromatosus (SLE) and the true incidence is unknown. This case report shares our experience with a 40 year old lady suffering from SLE whose Abdominal Aortic Aneurysm (AAA) was treated successfully with endovascular aneurysm repair (EVAR). To the author's knowledge, the use of EVAR for AAA in SLE patients has not been reported in available literature. The pathogenesis and differences in aneurysmal disease in patients with SLE is also discussed.
\end{abstract}

Keywords: Abdominal aortic aneurysm; Systemic lupus erythematosus; Endovascular repair aneurysm repair

\section{Introduction}

SLE is a chronic systemic inflammatory disease with multi-organ involvement including the cardiovascular system due to production of various auto-antibodies against a variety of auto-antigens. While hypertension, pericardial, myocardial as well as coronary artery disease are common manifestations, aortic aneurysm and dissection are much rarer but increasingly diagnosed. This could in part be due to the increased availability of non-invasive imaging techniques as well as the use of corticosteroids and other immune-modulators, suppressants or disease modifying anti-rheumatic drugs, which have led to the prolonged survival of patients with SLE. Little is known about the pathophysiology of aneurysms in these patients and their rate of diameter growth though they are generally presumed to be associated with inflammatory activity as well as the premature development of atherosclerosis. The use of immunosuppressant has also been thought to have a role in the pathogenesis of aneurysms by in these aspects. Likewise, the best treatment is not well defined and to the author's knowledge the use of EVAR for abdominal aortic aneurysms in SLE patients has not been reported in available literature. Inevitably, the suitability, graft durability and the need for lifelong surveillance in these patients remains uncertain. A young lady with a history of SLE on chronic steroids and other immunosuppressant who was incidentally found to have a palpable asymptomatic AAA is presented in this report. She underwent a successful EVAR and was followed up subsequently.

\section{Case Report}

A 40-year-old lady with a significant history of SLE diagnosed when she was 24-years-old when she presented with bilateral malar rash and lower limb swelling secondary to lupus nephritis. Autoimmune panel was positive for anti-nuclear antibody (ANA), ENA, anti-Smith, anti-RNP, anti-Ro, anti-double stranded DNA (DS DNA) and anticardiolipin IgG. She developed end stage renal failure after 2 years at 26-years-old, necessitating haemodialysis. Other complications of SLE that she suffers from include chronic haemolytic anaemia as well as hypertension. She also has an elder sister who also suffers from SLE and anti-phospholipid syndrome (APS) complicated by lupus nephritis and coronary arterial disease. Since her diagnosis, she has been treated with various immunosuppressants and disease modifying anti-rheumatic drugs (DMARDs) such as Cyclophosphamide, Prednisolone,
Azathioprine and Mycophenolate Mofetil. As a result, she has had drug related complications including thrombocytopenia, osteoporosis and CMV colitis, requiring multiple admissions.

\section{Diagnosis and investigations}

An incidental finding of an infra-renal AAA of $4.3 \mathrm{~cm}$ was first noted 3 years ago on an ultrasound examination of her abdomen for evaluation of her kidneys. During a subsequent follow up visit, a pulsatile mass was felt in her epigastrium. A Computed Tomography Aortogram (CTA) performed showed an infra-renal right-sided saccular aneurysm measuring $5.3 \times 4.8 \mathrm{~cm}, 4 \mathrm{~cm}$ inferior to the origin of the left renal artery (Figure 1). An eccentric rind of mural thrombus was seen within. Mild atherosclerotic calcification was seen in the abdominal aorta with no wall thickening to suggest aortitis. She was otherwise asymptomatic with no fever or pain. Inflammatory markers

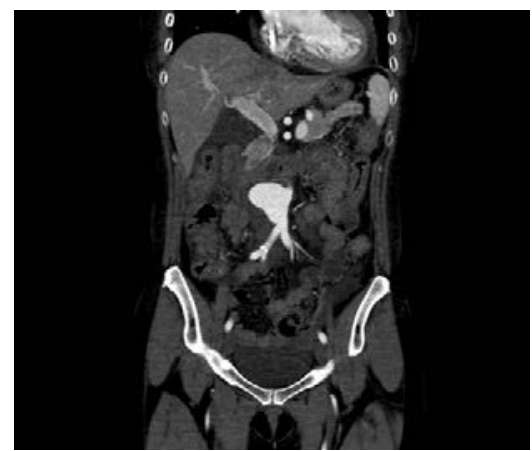

Figure 1: CT Aorta showing coronal and transverse views of saccular infrarenal AAA.

*Corresponding author: Nick Zhi Peng Ng, MBBS, MRCS, MMED, Singapore General Hospital, Singapore, Tel: +65 91524617; E-mail: ngzp_87@hotmail.com

Received June 07, 2017; Accepted June 26, 2017; Published June 29, 2017

Citation: Peng Ng NZ, Tze Tec C (2017) Endovascular Aneurysm Repair (EVAR) of an Infra-Renal Abdominal Aortic Aneurysm (AAA) in a Young Patient with Systemic Lupus Erythematosus (SLE). J Vasc Med Surg 5: 320. doi: 10.4172/23296925.1000320

Copyright: @ 2017 Peng Ng NZ, et al. This is an open-access article distributed under the terms of the Creative Commons Attribution License, which permits unrestricted use, distribution, and reproduction in any medium, provided the original author and source are credited. 
were not suggestive of an inflammatory or mycotic aneurysm, and blood cultures were negative for any bacterial growth. Her serum C-reactive protein (CRP) and Erythrocyte Sedimentation Ratio (ESR) trends did not exhibit a significant increase.

\section{Management}

She was offered a choice of open or endovascular repair of the AAA in view of her young age, and she opted for the endovascular approach. She underwent EVAR with the Medtronic Endurant II bifurcated stent graft. The procedure involved bilateral common femoral artery puncture under ultrasound guidance with the main body (ETBF 23$13 \mathrm{c} 124 \mathrm{EE})$ delivered via the right groin and the contralateral limb (ETLW 16-13c 82EE) through the left groin. The stent graft was then balloon moulded post deployment from the delivery device, and a check angiogram done showed satisfactory positioning with no type $1 / 3$ endoleak and a small type II endoleak (Figure 2) which was expected given that the IMA was patent preoperatively. Total operative time was an hour and fifteen minutes. Post-operatively, she was monitored in a high-dependency unit for 1 day, and was discharged well on the 3rd post-operative day. CT Aortogram performed at 1 and 6 months post EVAR showed that the graft continued to exclude the aneurysm sac, which measured $2.5 \mathrm{~cm}$ and $1.6 \mathrm{~cm}$, respectively (Figure 3) from $5.3 \mathrm{~cm}$ originally. A small and stable type II endoleak (likely from either inferior mesenteric artery or lumbar branches) persisted. Given the sac shrinkage, the type II leak was not felt to be significant and monitored. She was initially scheduled to receive a renal transplant. However, 4 months post EVAR, she developed chest pain with dyspnoea. A coronary angiogram done revealed triple vessel disease, and she underwent coronary artery bypass graft (CABG) surgery. This was complicated by pericardial tamponade from a slow oozing bleeder necessitating a relook sternotomy. Fortunately, she was discharged well and was reviewed at the vascular clinic 2 months later for CT surveillance of the AAA repair.

\section{Discussion}

An aneurysm is defined by the Society of Vascular Surgery and
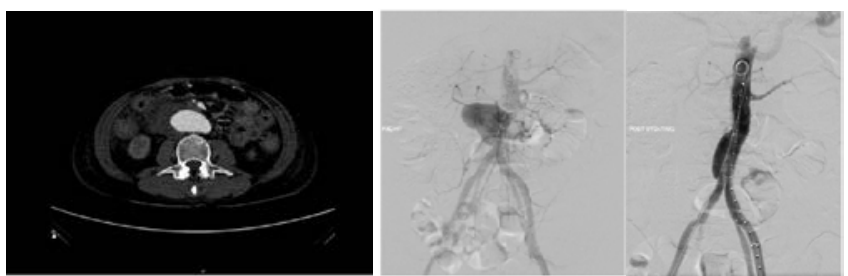

Figure 2: Angiography showing aortic pre and post stenting.
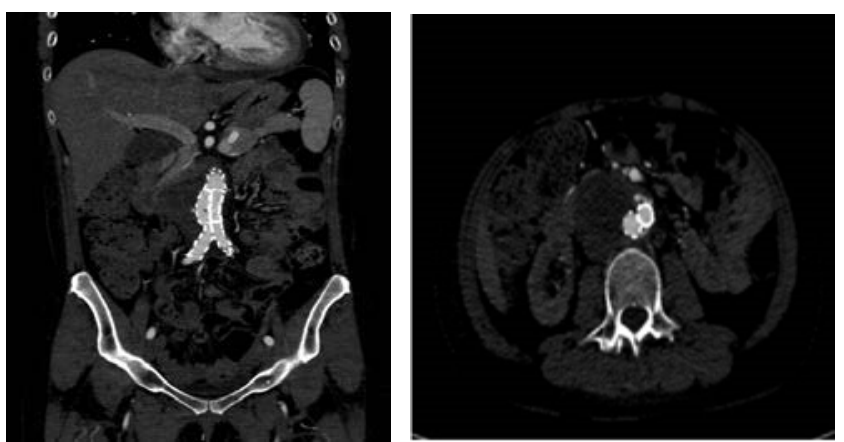

Figure 3: CT Aortogram 6 months post stent graft insertion. the International Society for Cardiovascular Surgery as a permanent localized dilatation of an artery having at least a $50 \%$ increase in diameter compared with the expected normal diameter of the artery or of the segment proximal.1 Key structural proteins such as elastin and collagen, as well as smooth muscle cells are arranged in concentric lamellae to withstand haemodynamic stresses in arteries. Loss of this architecture results from atheromatous degeneration of the intima and release of proteolytic enzymes from neutrophils within inflammatory infiltrate which affects all layers of the vessel. Further increased in wall stress brought about by hypertension enhances the growth rate of an aneurysm. In SLE, immunopathology results when polyclonal B cells produce pathogenic auto-antibodies which form immune complexes, depositing in sites such as the kidneys. SLE is also often associated with other autoimmune conditions, such as APS in $20-30 \%$. These patients suffer from coagulation defects, thrombocytopenia with thrombotic tendencies, affecting cerebral, renal and other vessels. Aortic aneurysms and dissection in SLE is rare but seen to affect patients at a relatively younger age in comparison to the general population with aortic aneurysms. The pathogenesis has been attributed to circulatory disturbances resulting from vasculitis and cystic medial degeneration as well as atherosclerosis. The occurrence of atherosclerosis is often considered to be due to long-term steroid treatment, hyperlipidemia, hypertension, and nephrotic syndrome [1] described his institution's experience with only 5 SLE patients in over 10 years of treating 429 patients with all aortic aneurysms. 2 Similar to her, 4 of the 5 patients had received long-term corticosteroid treatment with a mean of 23 years. This explains why the average age of these patients was almost 20 years younger than others without SLE. The age of other reported SLE with AAA cases likewise ranged from 28 to 65 years old (mean 40 years), which was about 30 years younger than the age of other AAA patients. Notably, other conditions requiring long term steroids have not been investigated to have an increased risk of AAA formation in the same way as SLE. Due to the greater prevalence of SLE in women, AAAs in SLE naturally have a female predominance, whilst AAA formation in general is more common in males. The location of aortic aneurysms in SLE was also reported to be highly variable, occurring in the ascending, arch and descending aorta both infra and supra-renally. Enlargement of the entire aorta was noted in rare cases. Kurata Atsushi [2] published a meta-analysis of published cases conducted to clarify characteristics that may contribute to aneurysm formation in SLE patients. 3 Relevant studies published from 1969 to 2008 were analysed and only 35 cases were identified. Significantly, he found that factors correlating with thoracic or abdominal aneurysms to differ and proposed 2 principal patterns, one being the fatal non-atherosclerotic thoracic aneurysm which was associated with cystic medial degeneration (CMD) and probably vasculitis and the other, the atherosclerotic abdominal aneurysm which was complicated by long-term steroid treatment showing a relatively favorable prognosis. Thoracic lesions showed a higher rate of death, while abdominal lesions were associated with a relatively favorable prognosis. He proposed that CMD in SLE occurs at a younger age and was closely associated with vasculitis rather than atherosclerosis, affecting the thoracic over the abdominal aorta. The vasculitic process is presumed to play a more crucial role in the pathogenesis of a thoracic aneurysm compared to artherosclerosis. Early recognition of aortic dissection is thus needed to perform timely surgical intervention and careful evaluation of patients with evidence of vasculitis and CMD especially in thoracic lesions. De Conti et al. [3] reported a ruptured descending thoracic aortic aneurysm in a 25-yearold female with SLE who underwent 19 years of steroid therapy. She was treated with 2 endovascular stent-grafts and discharged from hospital 13 days after the procedure in good health. 3 months later, she 
returned with hemorrhagic shock due an aortic-esophageal fistula. She underwent to an open emergency surgery, and died during the postoperative period. 2 other cases of successful endovascular stent-grafting of the descending thoracic aortic aneurysms have been reported but little is known about long-term durability of either device or repair for these patients. Abdominal lesions tend to be related to the duration of steroid therapy. Presumably, if steroid treatment is prolonged, development of atherosclerosis may facilitate the formation of aneurysm of the abdominal aorta, a major site of atherosclerosis. Similarly, other authors such as Washiyama et al. [4] proposed that aortic aneurysms in patients with SLE may be classified into aneurysms that are caused by inflammation per se or those resulting from atherosclerosis due to prolonged steroid therapy [5]. Hypertension was thought to be a further risk factor for acceleration of atherosclerosis and dissection or rupture [5], however, stated that "The patient who had SLE associated with vasculitis was often normotensive, while those without vasculitis had a history of hypertension," [2] meta-analysis, hypertension did not show a correlation with any factors except for a negative correlation with rupture and vasculitis. The data suggest that causes other than hypertension, including CMD presumably associated with vasculitis, may possibly play a more important role in the aneurysm rupture of SLE. Although formerly proposed that hypertension in patients with SLE should be controlled aggressively with low dose of steroids preferred to forestall the development of aortic aneurysm and dissection, the data suggests that hypertension in SLE is not a risk factor for rupture. There are only a handful of reports which describe detailed findings of these aneurysms histopathologically. A case report by Stehbens et al. [6] gave a pathological description of a non-dissecting aortic aneurysm with SLE. The findings of thrombi with underlying atheromatous debris containing lipophages and remaining medial elastic fibres deep to the debris, did not differ from findings in atherosclerotic AAA without SLE. While some cases reported intimal hypertrophy and medial atrophy with wide zone of destruction, this was not always consistent. In addition, the absence of necrotic microvasculitis accompanied by fibrinoid necrosis also reflects the effect of long-term steroid therapy, resulting in weakening of the medial elastic lamina in many aneurysms.

\section{Conclusion}

The precise pathogenesis and incidence of aortic aneurysm formation in SLE remains obscure. Indications for elective repair of AAA in patients with SLE likewise are aimed at avoiding a catastrophic rupture. It is possible that aneuryms associated with vasculitis and $\mathrm{CMD}$ are at higher risks of rupture and therefore would require surgery and smaller diameters. With few case reports, aortic dissection seems to take place at any range between diameters of 3 to $6 \mathrm{~cm}$. The presence of fibrinoid necrosis, a characteristic finding in the organs of patients with SLE, supports the causal link between prolonged corticosteroid therapy and accelerating atherosclerosis with aneurysm enlargement. Further studies are required to examine the link between SLE, long term use of immunosuppresants and AAA formation, as well as the suitability and durability of EVAR in AAA for SLE patients.

\section{References}

1. Ohara N, Miyata T, Kurata A, Oshiro H, Sato O, et al. (2000) Ten YearsExperience of Aortic Aneurysm Associated with Systemic Lupus Erythematosus. European Journal of Vascular and Endovascular Surgery 3: 288-293.

2. Kurata Atsushi (2010) Aortic aneurysms in systemic lupus erythematosus: a meta-analysis of 35 cases in the literature and two different pathogeneses. Cardiovascular Pathology 20: e1-e7.

3. De Conti DO, Dias RR, Fiorelli Al, Stolf NAG (2011) Ruptured thoracic aortic aneurysm in patient with systemic lupus erythematosus. Rev Bras Cir Cardiovasc 26.1: 128-130.

4. Washiyama N, Kazui T, Takinami M (2000) Surgical treatment of recurren abdominal aortic aneurysm in a patient with systemic lupus erythematosus. J Vasc Surg 32: 209-212.

5. Johnston KW, Rutherford RB, Tilson MD, Shah DM, Hollier L, et al. (1991) Suggested standards for reporting on arterial aneurysms. J Vasc Surg 13: 452-458.

6. Stehbens WE, Delahunt B, Shirer WC, Naik DK (1993) Aortic aneurysm in systemic lupus erythematosus. Histopathology, pp: 275-277. 